\title{
Mycobacterium Chlorophenolicum Isolation in Sirolimus-Associated Pneumonitis
}

\author{
Ramonell RP ${ }^{* 1}$, Kempker JA², Berkowitz EA ${ }^{3}$, Gal AA ${ }^{2}$ and Bag $\mathrm{R}^{4}$ \\ ${ }^{1}$ Department of Medicine, Emory University, Atlanta, Georgia, USA \\ ${ }^{2}$ Division of Pulmonary and Critical Care Medicine, Emory University, Atlanta, Georgia, USA \\ ${ }^{3}$ Department of Radiology and Imaging Sciences, Division of Cardiothoracic Radiology, Emory University School of \\ Medicine, Atlanta, Georgia, USA \\ ${ }^{4}$ Section of Pulmonary \& Critical Care Medicine, The University of Chicago Medicine, Chicago, Illinois, USA
}

${ }^{*}$ Corresponding author: Ramonell RP, Department of Medicine, Emory University, 49 Jesse Hill Jr. Dr. SE. \#490B Atlanta, Georgia, USA 30303, Fax: 888-339-2549, Tel: 561-317-5648, E-mail: rramone@emory.edu

Citation: Ramonell RP, Kempker JA, Berkowitz EA, Gal AA, Bag R (2016) Mycobacterium Chlorophenolicum Isolation in Sirolimus-Associated Pneumonitis. J Case Rep Stud 4(3): 304. doi: 10.15744/2348-9820.4.304

Received Date: March 13, 2016 Accepted Date: June 18, 2016 Published Date: June 21, 2016

\begin{abstract}
Mycobacterium chlorophenolicum is a nontuberculous mycobacterium that has previously been characterized as nonpathogenic. We describe a 44 year-old patient with a history of deceased donor renal transplant on sirolimus who was found to meet criteria for both sirolimus-associated pneumonitis and secondary infection with Mycobacterium chlorophenolicum. In addition to describing a new infection with a generally nonpathogenic organism, we believe this case could generate new hypotheses regarding nontuberculous mycobacteria and the pathogenesis of sirolimus-associated pneumonitis.
\end{abstract}

Keywords: Sirolimus; Mycobacterium chlorophenolicum; Sirolimus-associated pneumonitis; Renal transplant

\section{Introduction}

Sirolimus is a macrolide compound frequently used in solid organ transplantation for immunosuppression that has also been implicated in a pulmonary toxicity syndrome known as sirolimus-associated pneumonitis. Although sirolimus-associated pneumonitis has been more readily identified in recent years, the pathophysiology of this syndrome remains unknown. Theories to explain this phenomenon include dose-dependent drug toxicity and an autoimmune response to exposure of an occult pulmonary antigen $[1,2]$. In addition to predisposing patients to drug toxicity, sirolimus also predisposes patients to opportunistic infections. Here we present the first case of Mycobacterium chlorophenolicum grown from a lung tissue culture in a renal transplant patient with clinical and radiographic findings meeting criteria for both a nontuberculous mycobacterial pulmonary infection and sirolimusassociated pneumonitis. We use this illustrative case to discuss the implications of this finding as a true opportunistic infection, a contaminant, or as a hypothesis generating finding of the pathogenesis of sirolimus-associated pneumonitis.

\section{Case Presentation}

A 44 year-old male with a history of a deceased donor renal transplant for lupus nephritis at age 32 had been developing chronic allograft rejection secondary to interstitial fibrosis/tubular atrophy and chronic antibody-mediated rejection over the preceding four years. His course had been complicated by episodes of acute on chronic rejection necessitating several courses of high-dose prednisone. He was being evaluated for a second transplant when a routine CT of the chest without intravenous contrast showed diffuse 1 to $3 \mathrm{~mm}$ centrilobular nodules involving all lung lobes. A repeat high-resolution CT of the chest without intravenous contrast one month later showed significant interval progression of diffuse centrilobular nodules and tree-in-bud pattern throughout both lungs, more prominent in the upper lobes, concerning for endobronchial spread of infection (Figure 1).

The patient was seen in an outpatient pulmonary clinic one month later where he endorsed a six-month history of progressive dyspnea, low-grade fevers, malaise, and anorexia complicated by 25 -pound weight loss. He denied having a cough during this time but had started smoking marijuana regularly to help increase appetite. He reported adherence with his immunosuppressive regimen which included sirolimus $4 \mathrm{mg}$ daily, mycophenolate mofetil 1,000 mg twice daily, and prednisone $10 \mathrm{mg}$ daily. Three years prior to presentation, he was transitioned from a calcineurin-based immunosuppression to sirolimus due to calcineurin-induced nephrotoxicity. Other medications included hydrochlorothiazide, omeprazole, atorvastatin, nifedipine, and sodium bicarbonate. 

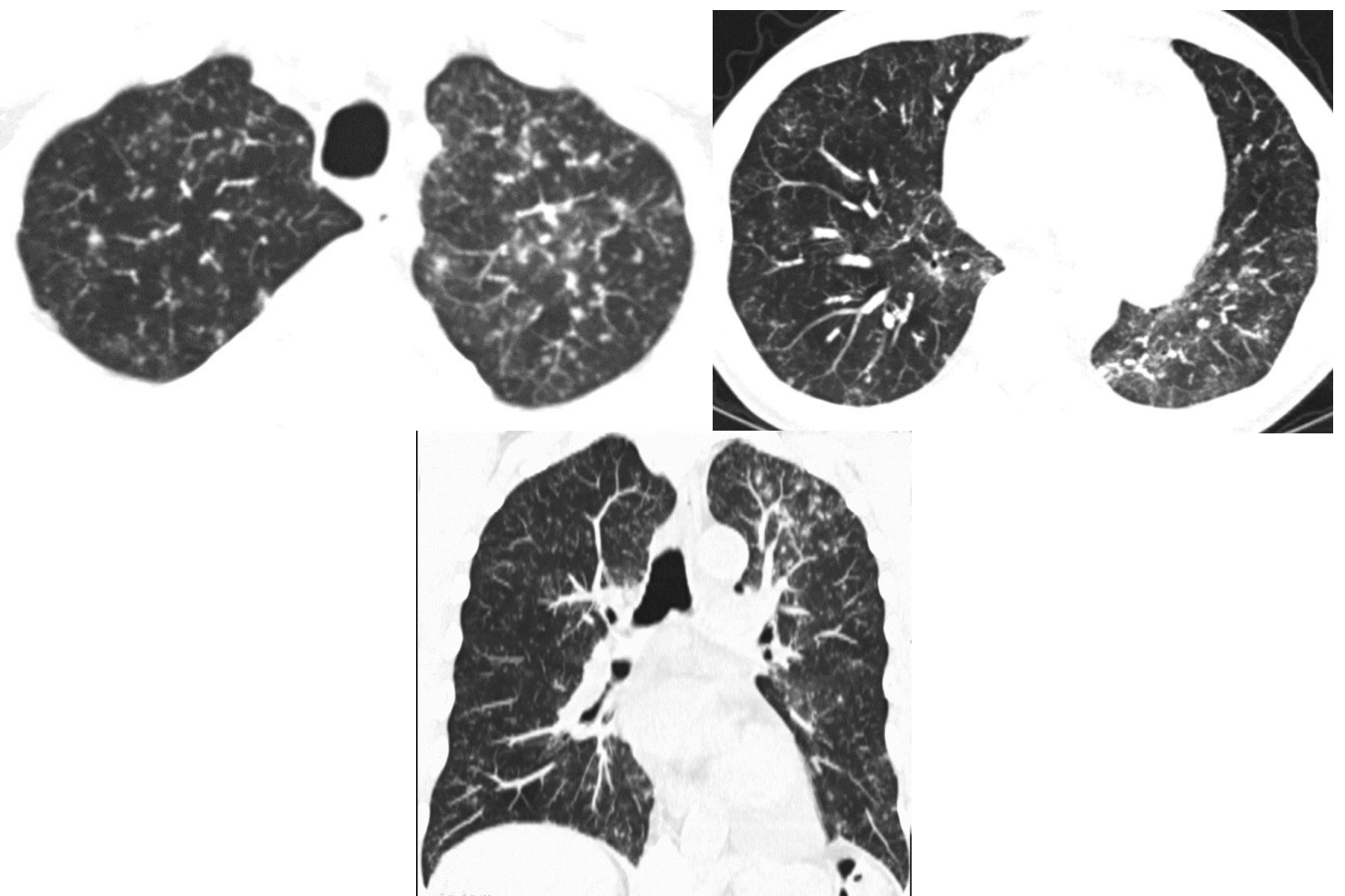

Figure 1: Axial and coronal CT chest images displayed in lung windows with Maximum Intensity Projection demonstrate diffuse centrilobular nodules $(1-3 \mathrm{~mm}$ ) with tree-in-bud pattern throughout the lungs with slight upper lung predominance

In clinic, the patient had a low-grade fever to 38.2 degrees Celsius, was tachycardic to 128 beats per minute, his oxygen saturation was $94 \%$ on room air, and he was ill appearing. His lungs were clear to auscultation and the rest of his examination was unremarkable. Initial labs were significant for blood urea nitrogen of $34 \mathrm{mg} / \mathrm{dL}$, creatinine of $3.8 \mathrm{mg} / \mathrm{dL}$, and hemoglobin of $8.2 \mathrm{~g} / \mathrm{dL}$. His white blood cell count was $7.9 \times 10^{9}$ cells/L with a differential of $78 \%$ neutrophils, $13 \%$ lymphocytes, and $1 \%$ eosinophils. His sirolimus trough was $5.7 \mathrm{ng} / \mathrm{dL}$ and had previously been maintained at his goal between 5 and $7 \mathrm{ng} / \mathrm{dL}$. The patient was admitted into the hospital and a chest CT without intravenous contrast showed interval worsening of diffuse ground glass and nodular opacities representing a progression of the disease process described on the chest CT one month prior (Figure 2).

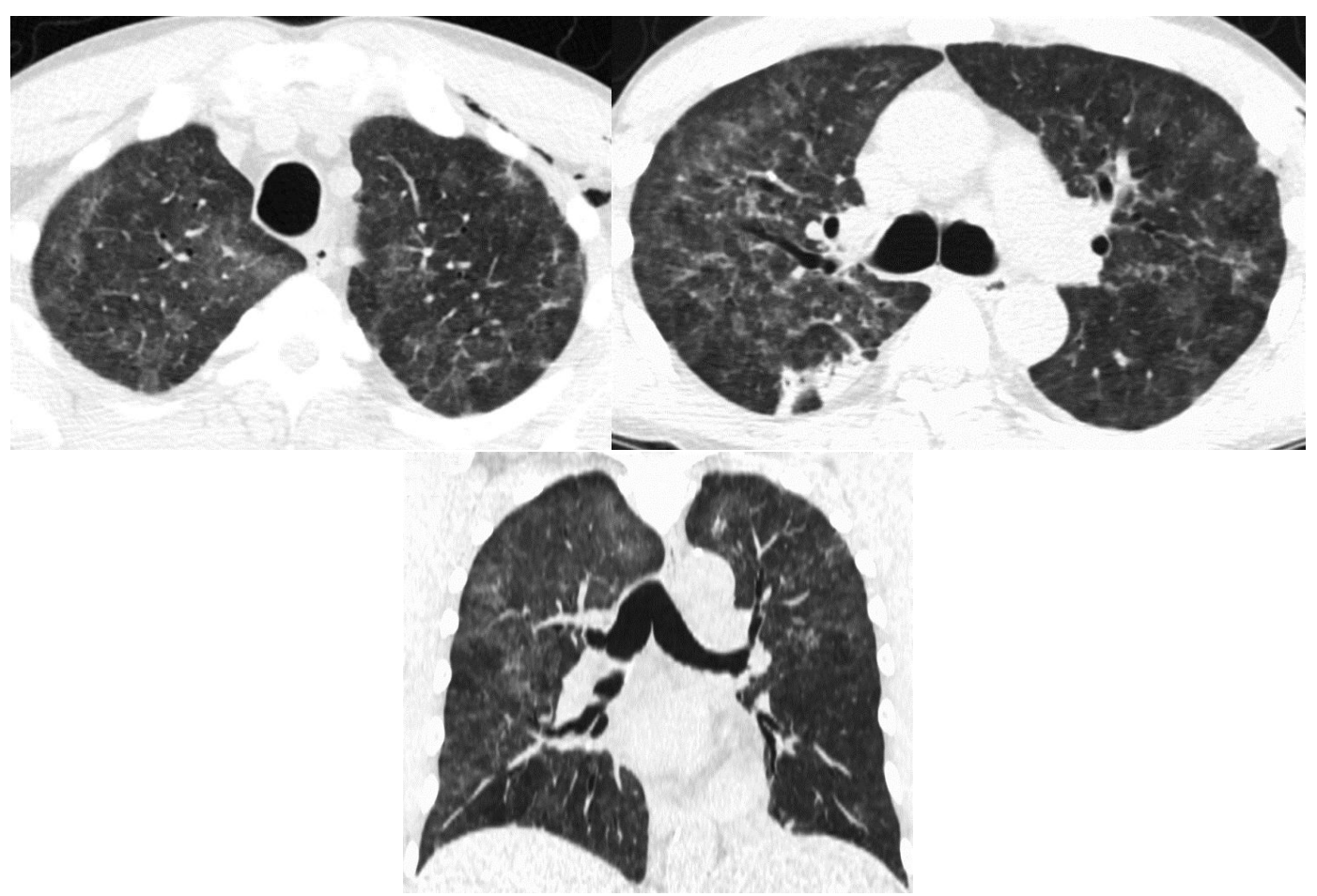

Figure 2: Axial and coronal CT chest images displayed in lung windows demonstrate patchy geographic ground glass opacities superimposed on decreasing diffuse centrilobular nodules $(1-3 \mathrm{~mm})$ and tree-in-bud pattern throughout the lungs. A subpleural right lower lobe consolidation represents organizing pneumonia 
A bronchoscopy with bronchoalveolar lavage (BAL) and transbronchial biopsy (TBB) was performed which demonstrated no endobronchial pathology and normal bronchial mucosa. Analysis of the BAL fluid sample showed 140 cells with a differential of 77\% lymphocytes, $8 \%$ macrophages, $6 \%$ neutrophils, $4 \%$ eosinophils, and 3\% siderophages. Cytology did not reveal malignant cells, and a Grocott's methenamine silver (GMS) stain was negative for Pneumocystis jirovecii. The TBB specimen showed loosely organized granulomas with clusters of intraalveolar macrophages (Figure 3). Stains for acid-fast bacilli (AFB) and fungi were negative. All fluid and tissue cultures were negative at one week. Sirolimus-associated pneumonitis was suspected and the medication was discontinued.

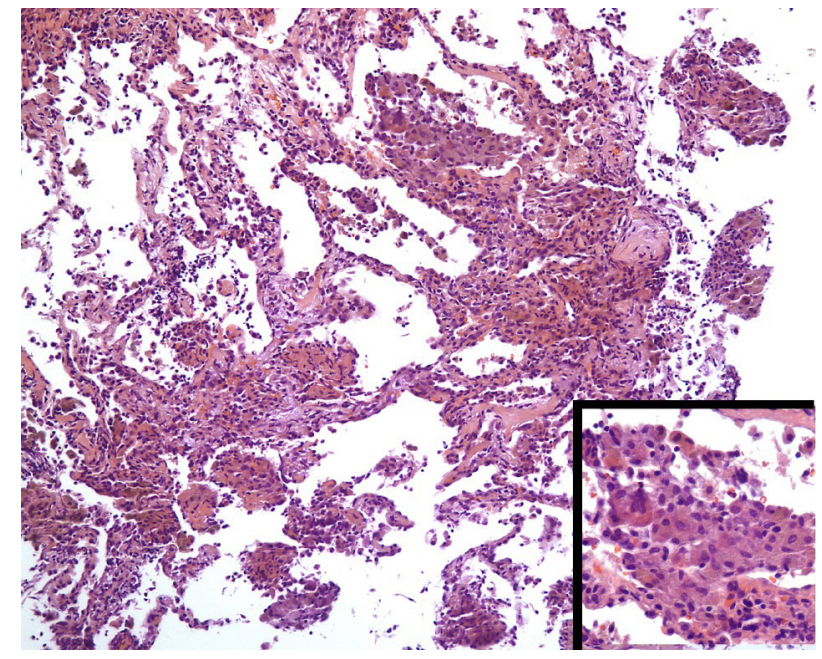

Figure 3: Hematoxylin and eosin staining of the transbronchial biopsy specimen (20X magnification with 40X magnification inset) shows vague granuloma formation with numerous clusters of intraalveolar macrophages. The inset shows a collection of intraalveolar macrophages and rare giant cells indicating a poorly formed granuloma

In the ensuing days, the patient's symptoms slowly improved. A CT chest without intravenous contrast on day seven of admission showed some resolution of the previously identified pulmonary nodules with development of diffuse ground glass opacities and a patchy consolidation in the posterior right lower lobe consistent with organizing pneumonia. At the time of hospital discharge, the patient was given a diagnosis of sirolimus-associated pneumonitis and prescribed a new immunosuppressive regimen including only mycophenolate mofetil 1,000 mg twice daily and prednisone $10 \mathrm{mg}$ daily. Notably, the patient had not received any antibiotics throughout the entire course of his illness.

One month after discharge, the patient's symptoms had significantly improved and a CT chest without intravenous contrast showed complete resolution of prior abnormalities. The AFB tissue culture from the patient's TBB specimen, which had been sent to the National Jewish Health Mycobacteriology Laboratory in Denver, Colorado, had grown Mycobacterium chlorophenolicum. Given his clinical and radiographic improvement, the patient was not initiated on antimycobacterial therapy.

\section{Discussion}

Sirolimus has been frequently used in solid organ transplantation for immunosuppression and as an alternative to calcineurin inhibitors in patients with renal dysfunction, as in this case. As its use spread, providers began to notice pulmonary toxicity and, in the early 2000s, the syndrome of sirolimus-associated pneumonitis was first described $[1,3]$. Although several theories behind the pathogenesis of this entity have been proposed in the literature, infectious causes are not believed to contribute to its development. In the present case, the patient meets criteria for both sirolimus-associated pneumonitis and a nontuberculous mycobacterial infection, raising the hypothesis that occult infection with a generally nonpathogenic organism could have resulted from interference in innate and adaptive immunity caused by sirolimus.

Sirolimus-associated pneumonitis in renal transplant recipients has been well described in the literature and is characterized as having symptoms of cough, fatigue, fever, and dyspnea following initiation of sirolimus that results in a bilateral interstitial infiltrate pattern on CT and lymphocyte-predominance on BAL [1,3-5]. Proposed criteria for the diagnosis of sirolimus-associated pneumonitis in renal transplant recipients have not been firmly established but include exposure to sirolimus preceding the onset of pulmonary symptoms, exclusion of infection or alternative pulmonary disease, resolution after sirolimus discontinuation, lymphocytic alveolar cellular profile on BAL, and organizing pneumonia pattern on CT of the chest $[1,4,6]$. Although the most common radiographic finding in sirolimus-associated pneumonitis is an organizing pneumonia pattern, other patterns have been reported including interstitial pneumonitis and alveolar hemorrhage [2].

A case series of 64 solid organ transplant recipients who developed pulmonary toxicity meeting criteria for sirolimus-associated pneumonitis demonstrated that risk factors for this condition include high dose of sirolimus independent of serum trough level, taking sirolimus as a second-line therapy (most commonly after calcineurin-inhibitor nephrotoxicity) and male gender [5]. In 
another case series of 24 renal transplant recipients who developed sirolimus-associated pneumonitis, the median exposure to sirolimus was 5.5 months before respiratory symptoms emerged. Most patients had normal resting oxygen saturation and hypercellularity in BAL fluid analysis with lymphocyte-predominance in 19 of the 24 patients. The majority of patients had an organizing pneumonia pattern on CT of the chest and no bacterial, viral, parasitic, or fungal organisms were identified on various microbiological evaluations. All patients recovered following withdrawal of sirolimus [4].

In addition to possible direct pulmonary toxicity, the decrease in $\mathrm{T}$ cell function caused by sirolimus may predispose transplant patients taking this medication to infections from nontuberculous mycobacteria (NTM) although the data supporting this hypothesis is limited [7]. According to a review by Doucette and Fishman, the incidence of NTM infections among patients who have received a renal transplant is estimated to be between $0.16 \%$ and $0.38 \%$. The majority of renal transplant patients who develop NTM infections will present with disseminated or localized cutaneous disease; disseminated disease is the second most common presentation [8].

In immunocompromised hosts, the diagnosis of NTM infection is often difficult to make. The majority of NTM organisms are ubiquitous in the environment and therefore laboratory isolation may represent contamination or colonization instead of a true infection [8]. For this reason, the American Thoracic Society and Infectious Diseases Society of America jointly published their third and most recent guidelines in 2007 that established a uniform set of criteria for the diagnosis of NTM infections. These guidelines state that the diagnosis of NTM pulmonary disease should be based on clinical criteria including pulmonary symptoms, nodular or cavitary opacities on chest radiography or multifocal bronchiectasis with multiple small nodules on high-resolution CT of the chest, and microbiologic criteria that require at least one of the following three conditions: 1) positive sputum cultures, 2) positive BAL or bronchial wash cultures, or 3) positive TBB tissue culture with histologic features consistent with mycobacterial infection [9].

Complicating the diagnosis of an NTM organism in an immunosuppressed host is the fact that the patient in this case smoked marijuana regularly. Marijuana has been associated with several impairments in immune function including deterioration in mucociliary clearance that, in this patient, could have further suppressed the function of his already compromised immune system [10]. In addition, marijuana was shown to be contaminated with bacteria, fungi, mycobacteria, and other soil dwelling-pathogens. Therefore, smoking marijuana could be the route of delivery of a multitude of pathogens, including soil-dwelling pathogens such as Mycobacterium chlorophenolicum [10].

Mycobacterium chlorophenolicum is an actinomycete which, like other members of the genus Mycobacterium, is ubiquitous in the environment. Originally named Rhodococcus chlorophenolicus, M. chlorophenolicum is most closely related to Mycobacterium chubuense and is generally regarded to be nonpathogenic although little is known about its potential for human disease $[11,12]$. In the report discussed above, our patient was found to meet criteria for both an NTM pulmonary infection with $M$. chlorophenolicum and sirolimus-associated pneumonitis. Although the guidelines for both sirolimus-associated pneumonitis and NTM pulmonary infection specify that all other diagnoses must be excluded, the possibility exists that our patient may have had either: 1) both an NTM infection and sirolimus-associated pneumonitis that were temporally related, 2) an NTM infection related to sirolimus immunosuppression manifesting as the syndrome of sirolimus-associated pneumonitis and resolving with partial immune reconstitution or 3) sirolimus-associated pneumonitis with contamination of the TBB tissue culture. Of note, this patient was receiving a low sirolimus dose not usually characteristic of those suffering from sirolimus-associated pneumonitis but other clinical and radiological findings support this diagnosis.

Sirolimus is used to prevent graft rejection in solid organ transplantation because of its effects on both innate and adaptive immunity $[13,14]$. Specifically, sirolimus binds to cytosolic FK binding protein 12 and forms a complex that then binds to and inhibits the mammalian target of rapamycin (mTOR) complex 1. Inhibition of the mTOR pathway disrupts interleukin 2 receptor signaling and causes cell cycle arrest in the G1 phase thus decreasing $\mathrm{T}$ cell proliferation [15]. The effect of mTOR inhibitors, such as sirolimus, on innate and adaptive immunity is complex. Sirolimus is believed to induce immunosuppression through inhibition of T lymphocyte proliferation while promoting immune tolerance through a preferential increase in CD4+CD25+Forkhead box P3+ regulatory $\mathrm{T}$ cell production [13,16-18]. Furthermore, mTOR inhibition triggers the production of anti-inflammatory cytokines and alters the ability of antigen-presenting cells to process intracellular antigens. Decreased intracellular antigen processing could attenuate the host's ability to fight pathogens and could allow the survival of mycobacteria like M. chlorophenolicum [19]. It is therefore plausible that these complex relationships led to an opportunistic infection with M. chlorophenolicum, which resolved upon cessation of mTOR inhibition.

Patients with sirolimus-associated pneumonitis have been shown to have a plurality of CD4-positive T cells in fluid from alveolar lavage, a pattern of adaptive immunity that would be expected with NTM infection [3-5,20,21]. Given that sirolimus preferentially mitigates CD4-positive T cell function, one may hypothesize that our patient could not mount an effective response to $M$. chlorophenolicum until his sirolimus was discontinued. Other case series of sirolimus-associated pneumonitis have not found $M$. chlorophenolicum on TBB however the majority of patients included in the literature underwent only BAL $[1,3,5]$. 
In summary, this case represents the first documented human infection with $M$. chlorophenolicum. Although significant investigation remains to be done into mechanisms of NTM infection and the pathogenesis of sirolimus-associated pneumonitis, this case emphasizes the possibility that sirolimus may predispose patients to infection with M. chlorophenolicum. We recognize that our case report is the only example of infection with an organism that is generally regarded to be nonpathogenic, but until a more concrete mechanism of sirolimus-associated lung injury emerges, we believe that opportunistic infection with $M$. chlorophenolicum and its relation to sirolimus-associated pneumonitis merits further scientific investigation.

\section{References}

1. Morelon E, Stern M, Israël-Biet D, Correas JM, Danel C, et al. (2001) Characteristics of sirolimus Associated Interstitial pneumonitis in renal transplant patients. Transplantation 72: 787-90.

2. Khalife WI, Kogoj P, Kar B (2007) Sirolimus-induced alveolar hemorrhage. J Heart Lung Transplant 26: 652-7.

3. Morelon E, Stern M, Kreis H (2000) Interstitial pneumonitis associated with sirolimus therapy in renal transplant recipients. N Engl J Med 343: 225-6.

4. Champion L, Stern M, Israël-Biet D, Mamzer-Bruneel MF, Peraldi MN, et al. (2006) Brief communication: sirolimus-associated pneumonitis: 24 cases in renal transplant recipients. Ann Intern Med 144: 505-9.

5. Garrean S, Massad MG, Tshibaka M, Hanhan Z, Caines AE, et al. (2005) Sirolimus-associated interstitial pneumonitis in solid organ transplant recipients. Clin Transplant 19: 698-703.

6. Banerjee SK, Santhanakrishnan K, Tsui S, Parameshwar J, Parmar J (2012) Cavitatory lung disease in thoracic transplant recipients receiving sirolimus. J Heart Lung Transplant 31: 548-51.

7. Knoll BM (2014) Update on nontuberculous mycobacterial infections in solid organ and hematopoietic stem cell transplant recipients. Curr Infect Dis Rep 16: 421.

8. Doucette K, Fishman JA (2004) Nontuberculous mycobacterial infection in hematopoietic stem cell and solid organ transplant recipients. Clin Infect Dis 38: 1428-39.

9. Griffith DE, Aksamit T, Brown-Elliott BA, Catanzaro A, Daley C, et al. (2007) An official ATS/IDSA statement: diagnosis, treatment, and prevention of nontuberculous mycobacterial diseases. Am J Respir Crit Care Med 175: 367-416.

10. Tashkin DP (2013) Effects of Marijuana Smoking on the Lung. Ann Am Thorac Soc 10: 239-47.

11. Wang Y, Ogawa M, Fukuda K, Miyamoto H, Taniguchi H (2006) Isolation and identification of mycobacteria from soils at an illegal dumping site and landfills in Japan. Microbiol Immunol 50: 513-24.

12. Häggblom MM, Nohynek LJ, Palleroni NJ, Kronqvist K, Nurmiaho-Lassila EL, et al. (1994) Transfer of polychlorophenol-degrading Rhodococcus chlorophenolicus (Apajalahti et al. 1986) to the genus Mycobacterium as Mycobacterium chlorophenolicum comb. nov. Int J Syst Bacteriol 44: 485-93.

13. Thomson AW, Turnquist HR, Raimondi G (2009) Immunoregulatory functions of mTOR inhibition. Nat Rev Immunol 9: 324-37.

14. Marx SO, Jayaraman T, Go LO, Marks AR (1995) Rapamycin-FKBP inhibits cell cycle regulators of proliferation in vascular smooth muscle cells. Circ Res 76: 412-7.

15. Dupont P, Warrens AN (2003) The evolving role of sirolimus in renal transplantation. QJM 96: 401-9.

16. Lin HY, Chang KT, Hung CC, Kuo CH, Hwang SJ, et al. (2014) Effects of the mTOR inhibitor rapamycin on monocyte-secreted chemokines. BMC Immunol 15: 37.

17. Li Y, Shi Y, Liao Y, Yan L, Zhang Q, et al. (2015) Differential regulation of Tregs and Th17/Th1 cells by a sirolimus-based regimen might be dependent on STATsignaling in renal transplant recipients. Int Immunopharmacol 28: 435-43.

18. Gallon L, Traitanon O, Yu Y, Shi B, Leventhal JR, et al. (2015) Differential Effects of Calcineurin and Mammalian Target of Rapamycin Inhibitors on Alloreactive Th1, Th17, and Regulatory T Cells. Transplantation 99: 1774-84.

19. Zullo AJ, Jurcic Smith KL, Lee S (2014) Mammalian target of Rapamycin inhibition and mycobacterial survival are uncoupled in murine macrophages. BMC Biochem 15: 4.

20. Huang LK, Tsai MJ, Chang SC (2013) Unusual radiological presentation of sirolimus-associated pneumonitis. J Chin Med Assoc 76: 588-92.

21. Howard L, Gopalan D, Griffiths M, Mahadeva R (2006) Sirolimus-induced pulmonary hypersensitivity associated with a CD4 T-cell infiltrate. Chest 129: $1718-21$.

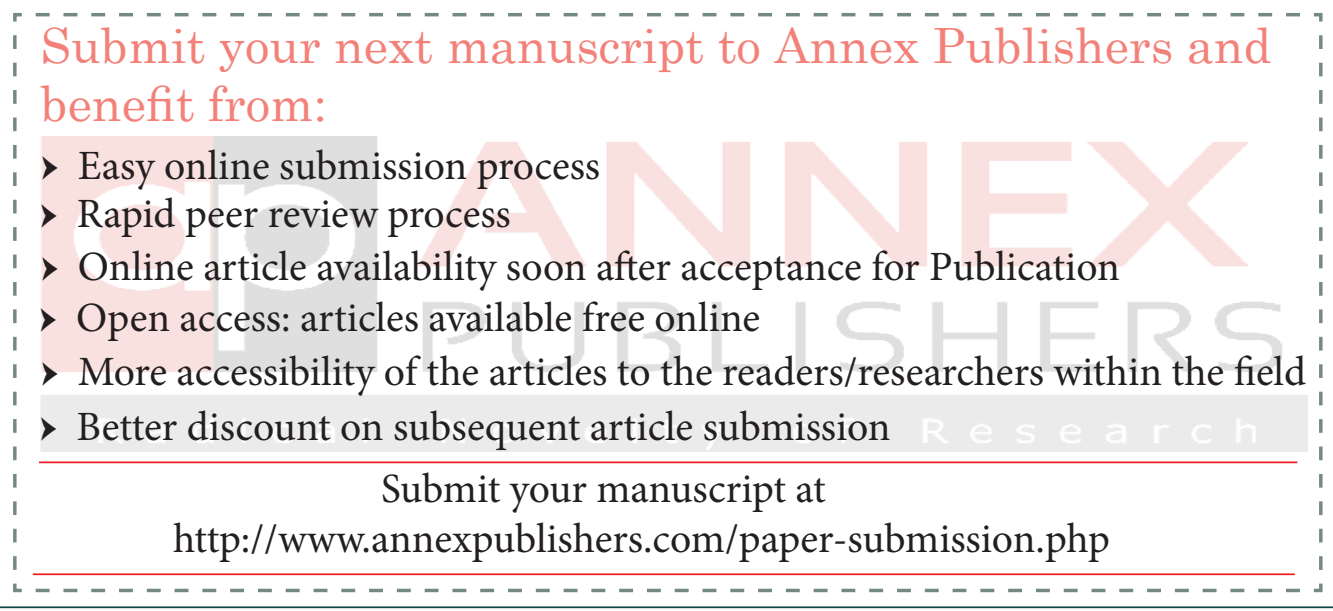

